\title{
齿科用氧化锆陶瓷韧性研究进展
}

\author{
朱东涁 $^{1,2}$, 宋艳军 ${ }^{2}$, 梁金生 ${ }^{2}$, 张晓旭 ${ }^{1}$, 楚锐清 ${ }^{1}$, 吴民强 ${ }^{1}$
}

(河北工业大学 1. 机械工程学院; 2. 生态环境与信息特种功能材料教育部重点实验室, 天津 300130)

\begin{abstract}
摘 要: 氧化锆陶瓷具有高强度、高韧性、高硬度、耐磨损、生物相容性好等优点, 广泛应用于齿科修复。但氧化 锆陶瓷相变增㓞会缩短其服役寿命, 尤其在极潮湿的口腔唾液等复杂的生物化学条件下, 因承受咀嚼力、温度的频 繁变化, 而导致其失效断裂。本文概述了氧化锆陶瓷在齿科修复领域的应用研究进展, 总结了氧化锆陶瓷的增韧机 理以及常用齿科氧化锆陶瓷的研究现状, 并对临床服役中氧化锆陶瓷的韧性老化现象进行分析, 总结了韧性老化 机理及其预防措施和方法。随着齿科氧化锆陶瓷综合力学性能的提高以及健康功能化的未来需求, 其在生物医用领 域的应用将会越来越广泛。
\end{abstract}

关 键 词: 氧化锆; 齿科; 韧性; 老化; 健康功能化; 综述

中图分类号: TQ174 文献标识码: A

\section{Progress of Toughness in Dental Zirconia Ceramics}

\author{
ZHU Dong-Bin ${ }^{1,2}$, SONG Yan-Jun², LIANG Jin-Sheng ${ }^{2}$, \\ ZHANG Xiao-Xu ${ }^{1}$, CHU Rui-Qing ${ }^{1}$, WU Min-Qiang ${ }^{1}$
}

(1. School of Mechanical Engineering, Hebei University of Technology, Tianjin 300130, China; 2. Key Laboratory of Special Functional Materials for Ecological Environment and Information of Ministry of Education, Hebei University of Technology, Tianjin 300130, China)

\begin{abstract}
Zirconia, attributable to high strength, toughness, hardness, and excellent wear resistance, as well as favorable biocompatibility, has been increasingly and widely applied to dental restoration. However, the phase transformation toughness of zirconia ceramics improves at the cost of service life deterioration. And zirconia ceramics may suffer failure and fracture after long-term mastication in the oral surroundings due to frequent changes in temperature and masticatory force, especially in the extremely complex biochemistry surroundings, such as fairly moist oral cavity and saliva. The research progress of zirconia ceramics in the field of dental restoration, toughening theories and their research state of dental ceramics were reviewed. This paper also summarized the issue of toughening degradation which is occurred in clinical application, and elucidated the mechanism and approaches. With the enhancement of comprehensive mechanical properties and the future demand for healthy functionalization, zirconia ceramics will be more widely applied in the biomedical field.
\end{abstract}

Key words: zirconia; dentistry; toughening; degradation; healthy functionalization; review

氧化锆 $\left(\mathrm{ZrO}_{2}\right)$ 陶瓷具有优良的力学性能、生物 相容性、美学性能等，被广泛应用于义齿修复及髋 关节替换 ${ }^{[1-2]}$ 。特别是 Garvie 等 ${ }^{[3]}$ 发现四方相 $\mathrm{t} \rightarrow$ 单 斜相 $\mathrm{m}$ 的相变可提高氧化锆陶瓷的断裂韧性以来,
它在生物医用领域的应用更加广泛。

氧化锆有三种同素异构体：单斜相(monoclinic, $\mathrm{P} 2_{1} / \mathrm{c}$ )、四方相(tetragonal, $\mathrm{P} 4_{2}-\mathrm{nmc}$ )、立方相(cubic, $\mathrm{Fm} \overline{3} \mathrm{~m})$ 。三者在不同温度下会发生晶型转变, 其中

收稿日期：2017-06-05; 收到修改稿日期：2017-09-11

基金项目: 河北省自然科学基金(E2013202128, E2018202200) Natural Science Foundation of Hebei Province (E2013202128, E2018202200)

作者简介: 朱东彬(1975-), 男, 博士, 副教授. E-mail: zhudongbin@hebut.edu.cn 
$950^{\circ} \mathrm{C}$ 左右降温时存在 $\mathrm{t} \rightarrow \mathrm{m}$ 马氏体相变, 会产生 3\% 5\%体积膨胀及 $1 \% \sim 7 \%$ 剪切应变 ${ }^{[4]}$ 。如果 $\mathrm{t}-\mathrm{ZrO}_{2}$ 在室温能够稳定存在, 就可利用 $\mathrm{t} \rightarrow \mathrm{m}$ 马氏体相变来 提高氧化锆陶瓷的韧性 ${ }^{[5-6]}$ 。通常可通过加入 $\mathrm{Y}_{2} \mathrm{O}_{3}$ 、 $\mathrm{CeO}_{2} 、 \mathrm{CaO} 、 \mathrm{MgO}$ 等稳定剂的方法来使 $\mathrm{t}-\mathrm{ZrO}_{2}$ 保存 到室温并稳定存在 ${ }^{[7]}$ 。

1969 年, Helmer 等首次将氧化锆陶瓷应用于生 物医用领域, 可替代氧化铝、金属钛等股骨头修复 体 ${ }^{[8]}$ 。氧化锆在骨科的研究主要考虑氧化锆的力学 性能、耐磨性及生物相容性 ${ }^{[9]}$ 。陶瓷在齿科中的应用 可以追溯到 1774 年, 法国药剂师 Duchatean 及牙科 医生 De Chemant 制作出世界上第一颗陶瓷义齿, 但 此陶瓷义齿韧性较差; 1886 年 Land 首次采用铂箔基 底烧制出瓷嵌体和瓷全冠, 1956 年后此项技术得到 进一步发展, 成为比较成熟的金属烤瓷牙技术 ${ }^{[10-11]}$ 。 此技术完美结合了金属的高强度与瓷的天然色泽, 但如果金属与陶瓷结合不良易导致崩瓷, 长期使用 个别金属还会渗出有毒物质。

近年来, 金属烤瓷牙技术逐渐被氧化锆全冠技 术所取代。20世纪 90 年代初, 氧化锆全冠开始采用 具有高度柔性的计算机辅助设计/计算机辅助制造 (CAD/CAM) 技术加工成形, 然后利用饰面长石瓷 进一步提高其美学性能 ${ }^{[12-13]}$ 。氧化锆全冠具有优异 的生物相容性及力学性能, 色泽自然、逼真。

然而, 在研磨或喷砂等加工过程中, 由于 $\mathrm{t}-\mathrm{ZrO}_{2}$ 的亚稳定性使陶瓷表面易发生 $\mathrm{t} \rightarrow \mathrm{m}$ 应力诱导相变, 虽然 $\mathrm{t} \rightarrow \mathrm{m}$ 相变可以提高氧化锆陶瓷的强度及韧性, 但同时因形成微裂纹而导致老化, 尤其是氧化锆陶 瓷处于潮湿的空气、水或水蒸气的服役环境中更易 诱发老化 ${ }^{[14-15]}$ 。研究表明氧化锆的低温老化的温度 范围在室温至 $400^{\circ} \mathrm{C}$ 左右, 很明显人体温度 $37^{\circ} \mathrm{C}$ 也 在这个范围 ${ }^{[16]}$ 。氧化锆陶瓷应力诱导相变对水及温 度的敏感性, 使其服役力学性能明显下降, 这也是 齿科用氧化锆陶瓷目前急需解决的问题。

本文从氧化锆陶瓷的增韧机制出发, 总结分析 了常用齿科氧化锆基陶瓷, 并概述了齿科氧化锆陶 瓷的韧性老化机理及防老化措施。最后, 对健康功 能化氧化锆陶瓷在生物医用领域的应用进行了展望。

\section{1 氧化锆陶瓷增韧机制}

\section{1 相变条件}

氧化锆陶瓷 $\mathrm{t} \rightarrow \mathrm{m}$ 相变对温度、应力变化比较敏 感, 相变晶粒也会受到整个体系自由能的影响。相变 时单独晶粒的体系自由能的变化由公式(1) ${ }^{[17]}$ 表示:
$\Delta G=-V_{\mathrm{p}} \Delta G 0+\Delta V=-V_{\mathrm{p}} \Delta G 0-\Delta V_{\mathrm{p}} e_{i j}^{\mathrm{T}}\left[P_{i j}^{\mathrm{A}}+\left(\frac{1}{2}\right) P_{i j}^{i}\right](1)$

$\Delta G_{0}$ 一相变与未相变的每摩尔化学能的自由能差;

$V_{\mathrm{p}}$ 一晶粒体积;

$P_{i j}^{\mathrm{A}} 、 P_{i j}^{\mathrm{i}}$ 一施加应力、晶粒相变时的应力;

$e_{i j}^{\mathrm{T}}$ 一无约束应力时的应变;

$P_{i j} 、 e_{i j}$ 一作用于外法线沿 $i$ 轴平面, $j$ 轴方向的应力 应变。

该公式适用于材料被施加应力的情况, 从热力 学角度 $(\Delta G \leqslant 0)$ 得出相变所需的应力必须满足以下 条件:

$$
P_{i j}^{\mathrm{A}} e_{i j}^{\mathrm{T}} \geqslant-P_{i j}^{\mathrm{i}} e_{i j}^{\mathrm{T}} / 2-\Delta G_{0}
$$

处于压应力状态的微裂纹尖端的相变晶粒不同 程度偏离其原来平衡位置后处于弹性应变状态, 引 起能量的升高并产生内应力, 应力 $P_{\mathrm{ij}}^{\mathrm{A}}$ 可通过平面应 变弹力场公式获得:

$$
\begin{array}{r}
\left.\begin{array}{c}
P_{y y}^{\mathrm{A}} \\
P_{x x}^{\mathrm{A}} \\
P_{x y} \mathrm{~A}
\end{array}\right\}=\frac{K_{\mathrm{I}} \cos \left(\frac{\theta}{2}\right)}{\sqrt{2 \pi r}}\left\{\begin{array}{l}
1-\sin \left(\frac{\theta}{2}\right) \sin \left(\frac{3 \theta}{2}\right) \\
1+\sin \left(\frac{\theta}{2}\right) \sin \left(\frac{3 \theta}{2}\right) \\
\sin \left(\frac{\theta}{2}\right) \sin \left(\frac{3 \theta}{2}\right)
\end{array}\right\} \\
P_{z z}^{\mathrm{A}}=v\left(P_{x x}^{\mathrm{A}}+P_{y y}^{\mathrm{A}}\right) \quad(r \leqslant a)
\end{array}
$$

式中 $K_{\mathrm{I}}$ 一应力强度因子;

$v$ 一泊松比;

$r$ 一距裂纹尖端的距离;

$a$ 一裂纹长度;

$\theta$ 一裂纹平面的角坐标;

将公式(3)、(4)代入公式(2)后所得的结果即是位于裂 纹尖端单个晶粒相变所需要的能量, 只有大于该值 相变才会发生。

\section{2 增韧机理}

与金属相比, 陶瓷材料的断裂韧性通常低 1 2 数量级。氧化锆陶瓷可以通过不同的增韧方式来提 高其断裂韧性, 主要增韧机理有: 应力诱导相变增 韧、微裂纹增韧、微裂纹的弯曲、分叉与桥接增韧、 晶须增韧、弥散增韧、细晶强化、纤维增韧等, 在 实际应用中氧化锆陶瓷材料韧性的提高往往是多种 增韧机制共同作用的结果。目前实验室测量氧化锆 陶瓷断裂韧性应用最多的方法有：单边切口梁法 (SENB)和压痕法(IM)。

氧化锆陶瓷韧性的研究早在 20 世纪 50 年代就 已经开始, 1975 年之后随着相变现象被发现, 一些 研究者 ${ }^{[5,18]}$ 认为应力诱导相变增韧是氧化锆陶瓷因 
外应力作用而产生裂纹, 裂纹尖端的应力能够诱发 $\mathrm{t} \rightarrow \mathrm{m}$ 马氏体相变(如图 1 所示为应力作用下发生的 $\mathrm{t} \rightarrow \mathrm{m}$ 相变过程), 相变晶粒产生的体积膨胀会抑制 裂纹扩展, 从而提高材料的韧性。但是, 在相变初始 阶段, 裂纹尖端 $120^{\circ}$ 夹角内存在的膨胀变形会引起 $\mathrm{ZrO}_{2}$ 韧性降低, 如图 2 所示, 之后体积膨胀会抑制 裂纹扩展, 使韧性迅速提高, 当裂纹扩展至 $5 \sim 10 \mathrm{~h}$ 时，断裂㓞性增长缓慢 ${ }^{[19]}$ 。

Mcmeeking 等 ${ }^{[20]}$ 首次提出残余应变场会诱发相 变并抑制微裂纹扩展从而提高材料㓞性，可以通过 微裂纹尖端附近的颗粒变形造成应力集中程度来评
估㓞性提高大小。若想应力诱导相变增韧机制发挥 作用, 则需要：1)氧化锆粒径尺寸须小于临界相变尺 寸; 2)外加作用力须大于相变形核所需势垒(2.1 内容); 3)基体材料对氧化锆具有约束力。同时相变量的增 加也成为评估韧性提高的另一种方法, Amazigo 等 ${ }^{[21]}$ 认为相变强度 $w$ 和相变体积含量 $V_{\mathrm{f}}$ 具有以下函数关系:

$$
w=\frac{1+v}{1-v}\left(\frac{E V_{\mathrm{f}} \theta_{\mathrm{p}}}{E_{\mathrm{m}}}\right)
$$

其中, $v 、 E$ 是泊松比及弹性模量, $\theta_{\mathrm{p}}$ 和 $E_{\mathrm{m}}$ 是净应变 和相变诱导临界应力。可见相变含量越多、相变诱 导临界应力越小，相变强度越高。
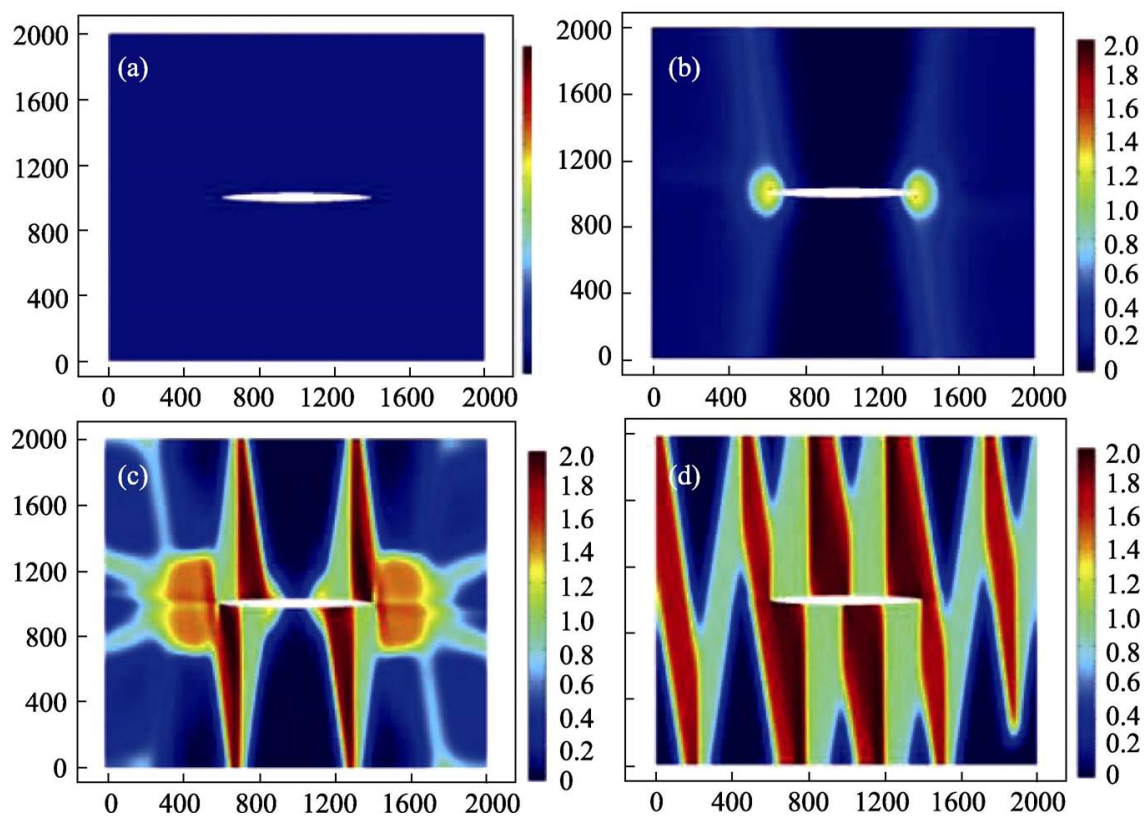

图 1 应力诱导 $\mathrm{t} \rightarrow \mathrm{m}$ 相变过程 ${ }^{[6}$

Fig. 1 Nucleation and evolution of monoclinic phase in a cracked tetragonal single crystal under tension stress ${ }^{[6]}$ (a)-(d): Correspond to time 0, $1.4 \mu \mathrm{s}, 1.6 \mu \mathrm{s}, 2.5 \mu \mathrm{s}$, respectively

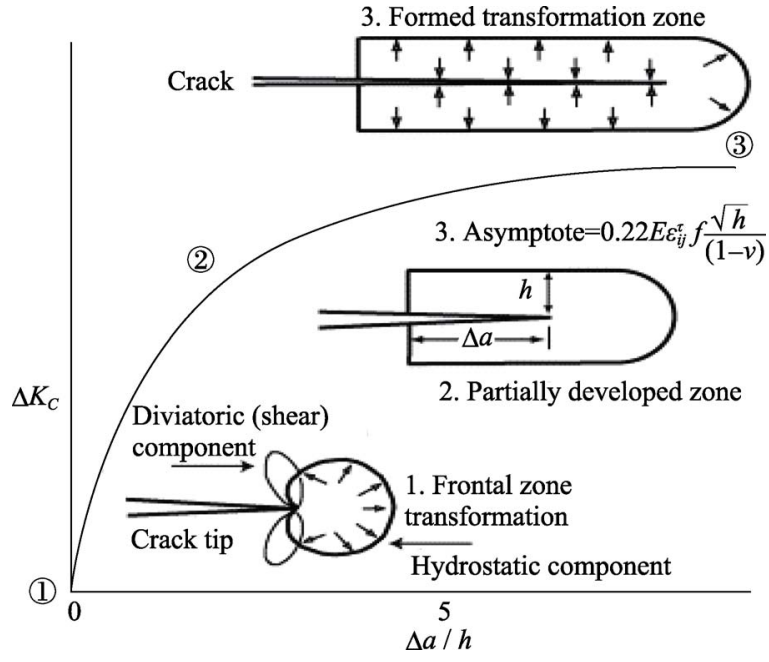

图 2 相变区中裂纹扩展伴随韧性提高的示意图 ${ }^{[19]}$

Fig. 2 Schematic illustration of transformation zone and toughness increment developed with crack extension ${ }^{[19]}$
在外力作用下，氧化锆陶瓷材料既会诱发主裂 纹外, 还会产生微裂纹, Griffth 微裂纹强度理论认 为物体内储存的弹性应变能的降低是裂纹扩展的动 力, 因此微裂纹的产生会降低相变区域的弹性模量, 分散和吸收主裂纹产生的能量从而提高材料的断裂 能起到增韧效果。Zhao 等 ${ }^{[22]}$ 利用双相场模型模拟相 变及裂纹扩展过程，如图 3 所示 $0 、 1.2 、 4.8 、 10 \mu \mathrm{s}$ 时裂纹扩展情况, 相变释放了裂纹尖端的应力, 相 变发生后裂纹扩展速率降低。由于相变后 $\mathrm{m}$ 相与变 形区交界处应力重新分配，微裂纹尖端会很快闭合。 Mamivand 等 ${ }^{[6]}$ 认为相变后裂纹尖端的晶粒优先在 裂纹表面产生, 导致裂纹表面发生 $\mathrm{t} \rightarrow \mathrm{m}$ 相变, 吸收 表面因膨胀而产生的应力能，从而引起微裂纹闭合。

氧化锆陶瓷微裂纹弯曲、分叉和桥接增韧是在 材料显微结构中通过人为控制或放置一些障碍物阻 


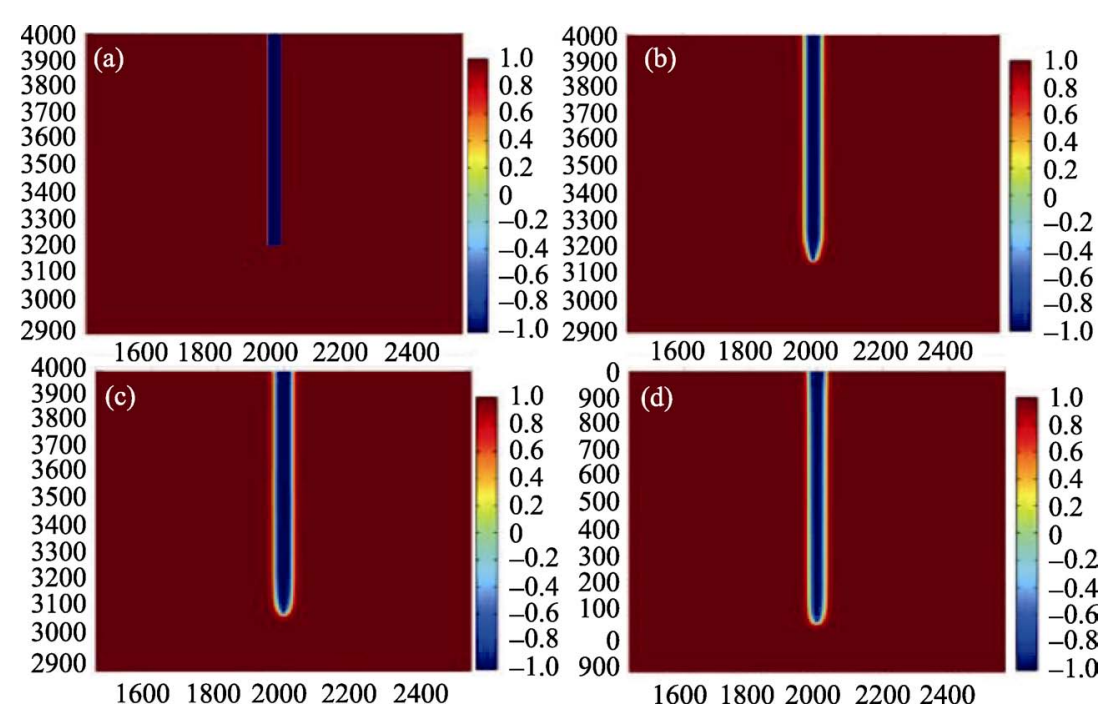

图 3 裂纹扩展过程 ${ }^{[22]}$

Fig. 3 Magnified view of the crack evolution ${ }^{[22]}$

(a)-(d): Correspond to time 0, $1.2 \mu \mathrm{s}, 4.8 \mu \mathrm{s}, 10.0 \mu \mathrm{s}$, respectively

碍裂纹的运动，使裂纹扩展过程中必须改变方向绕 过障碍物才能够继续进行, 增加裂纹扩展路径, 从 而达到增强增韧的目的。Smirnov 等 ${ }^{[23]}$ 通过放电等 离子体烧结工艺制备出 $\mathrm{ZrO}_{2} / \mathrm{Ta}$ 金属陶瓷复合材料, 如图 4 所示, 具有延展性的薄片金属 $\mathrm{Ta}$ 在材料断裂 时不仅可以吸收裂纹尖端的能量, 而且可以使裂纹 出现偏转和桥接、裂纹尖端应力松弛现象，同时 $\mathrm{Ta}_{2} \mathrm{O}_{5}$ 的存在也会促使 $\mathrm{ZrO}_{2}$ 发生相变, 使氧化锆的 断裂韧性提高了 $56 \%$ 。

晶须增韧是利用晶须高弹性模量、高强度优点来 分散基体外加应力, 晶须钉扎住裂纹后会阻止裂纹的 扩展，从而提高材料的韧性。具有较大长径比的晶须 为裂纹的偏转提供更长的路径, 并抑制晶粒的迁移 以及界面纤维拔出，从而更有助于提高材料的韧性。 $\mathrm{Liu}$ 等 ${ }^{[24]}$ 采用快速烧结法制备 $\mathrm{SiC}_{\mathrm{w}} / \mathrm{ZrO}_{2}$ 陶瓷, $\mathrm{SiC}$ 晶 须均匀分布在基体中并与基体形成紧密结合的锯齿 形态，如图 5(a)所示。为了解决 $\mathrm{SiC}$ 晶须增韧容易氧 化问题, Robertson 等 ${ }^{[25]}$ 采用莫来石(Mullite)纤维作为

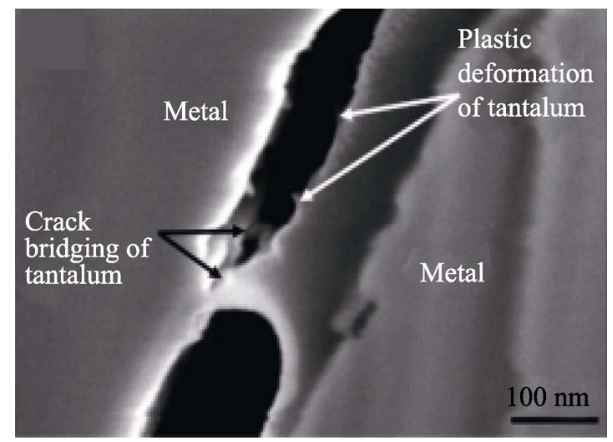

图 $4 \mathrm{ZrO}_{2} / \mathrm{Ta}$ 裂纹扩展中存在的微裂纹桥接 ${ }^{[23]}$

Fig. 4 Crack bridging occurs during crack propagation in zirconia-tantalum composite ${ }^{[23]}$
增韧材料，制备 Mullite $_{\mathrm{w}} / \mathrm{ZTA}$ 陶瓷，如图 5(b)所示氧 化铝晶粒间夹杂的 Mullite 晶须可抑制裂纹的扩展。

弥散强化增韧中弥散物质一般是硬质颗粒，材 料中出现的位错会绕过或切过第二相粒子, 同时晶 粒周围的弹性应力场与位错产生交互作用从而提高 其㓞性。例如 Pecharromán 等 ${ }^{[26]}$ 认为促使 $\mathrm{Ni} / \mathrm{ZrO}_{2}$ 复相陶瓷韧性、强度提高的主要原因是 $\mathrm{Ni}$ 合金颗粒 弥散和均匀分布。

连续纤维增韧是优于颗粒、晶须、层状增韧陶 瓷基复合材料的一种增韧方式 ${ }^{[27]}$ 。Dusza 等 ${ }^{[28]}$ 采用 放电等离子烧结工艺和热压烧结工艺制备碳纳米纤 维 $\mathrm{CNF} / \mathrm{ZrO}_{2}$ 陶瓷，研究发现 $\mathrm{CNF}$ 含量为 $2 \mathrm{wt} \%$ 时陶 瓷断裂韧性的最大值为 $6.4 \mathrm{MPa} \cdot \mathrm{m}^{1 / 2}$, 而纤维的拔 出、桥接、偏转(图 6 所示)及细晶强化是其断裂韧 性提高的主要原因。

对于氧化锆陶瓷材料, $\mathrm{t} \rightarrow \mathrm{m}$ 的相变量、晶粒尺 寸都是影响其力学性能的关键因素, 当相变量较少 时, 晶粒尺寸是韧性、强度提高的主导性因素, 晶粒
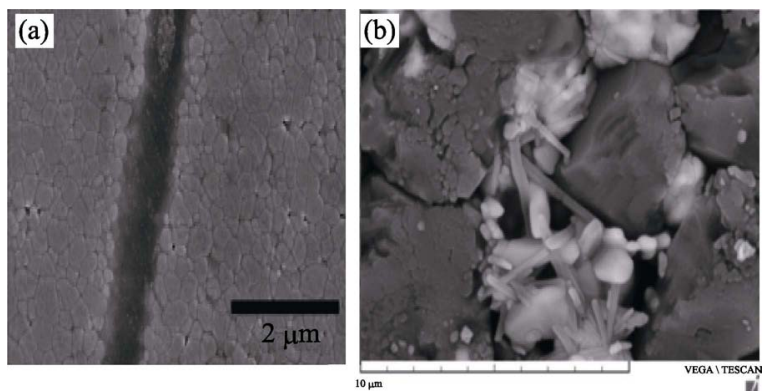

图 5 (a) $\mathrm{SiC}$ 晶须增㓞 $\mathrm{ZrO}_{2}$ 表面形貌 ${ }^{[24]}$ 和(b)Mullite 晶须增 韧 ZTA 表面形貌 ${ }^{[25]}$

Fig. 5 Morphologies of whisker reinforced zirconia ceramics (a) $\mathrm{SiCw} / \mathrm{ZrO}_{2}{ }^{[24]}$ and (b) Mullite ${ }_{\mathrm{w}} / \mathrm{ZTA}^{[25]}$ 


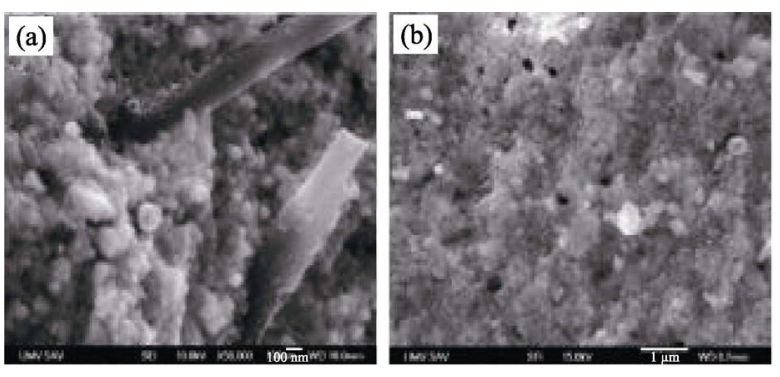

图 6 (a) 放电等离子烧结和(b) 热压烧结工艺形成的 $\mathrm{CNF} / \mathrm{ZrO}_{2}$ 断面形貌 ${ }^{[28]}$

Fig. 6 Fracture morphology of $\mathrm{CNF} / \mathrm{ZrO}_{2}$ sintered by (a) spark plasma sintering and (b) hot sintering ${ }^{[28]}$

尺寸越小, 晶界上原子排列不规则, 杂质缺陷多, 能量较高, 变形均匀, 应力集中小, 裂纹也不易萌 生及传播, 因此可起到增㓞效果。

\section{2 齿科氧化锆陶瓷}

目前齿科修复主要采用瓷熔附金属修复体(PFM) 和全瓷冠修复体。PFM 强度高, 但如果金属与陶瓷 结合不良, 容易出现崩瓷现象, 而且某些 PFM长期使 用可能溶出有毒物质, 如普遍使用的 Ni-Cr 合金, 因 而影响其长期临床使用。全瓷冠以其良好的生物相容 性及美学性受到广大研究者和患者青睐 ${ }^{[29]}$ 。氧化锆基 陶瓷因高强度、高韧性等特点使其在齿科用陶瓷中 脱颖而出。目前应用于齿科修复领域的氧化锆基陶瓷 主要有: $3 \mathrm{~mol} \% \mathrm{Y}_{2} \mathrm{O}_{3}$ 作为稳定剂的四方相多晶 $\mathrm{ZrO}_{2}$ 陶 瓷(3Y-TZP)、玻璃亲润陶瓷(In-Ceram)和氧化镁部分 稳定氧化锆(Mg-PSZ)。

\subsection{Y-TZP}

1976 年, Rieth 等首次发现用 $\mathrm{Y}_{2} \mathrm{O}_{3}$ 作稳定剂可 以使高温稳定的 $\mathrm{t}-\mathrm{ZrO}_{2}$ 在室温下稳定或亚稳定存 在, 之后通过实验证明含 $2 \mathrm{~mol} \%$ 3mol\% $\mathrm{Y}_{2} \mathrm{O}_{3}$ 稳定 $\mathrm{ZrO}_{2}$ 可以形成尺寸为数百纳米、具有完全四方相 的 TZP 陶瓷 ${ }^{[30]}$ 。8 80 年代后期 3Y-TZP 开始成为髋 关节假体中股骨的替代品, 与其它齿科陶瓷相比, 3Y-TZP 的抗弯强度(900 1400 MPa)和断裂韧性(9 $\left.20 \mathrm{MPa} \cdot \mathrm{m}^{1 / 2}\right)^{[31]}$, 该值远超咀嚼过程中受到的最大 咬合负荷, 已被应用于种植体-基台连接(Implant Ambutments)、牙齿修复(Teeth Restorations)及固定部 分牙齿(Fixed Partial Dentures, FPDs) ${ }^{[32]}$ 。

3Y-TZP 陶瓷最大的特点是具有应力诱导相变 增韧机制，但其表面四方相颗粒是不受基体控制的， 它可以自发转化为单斜相或者通过在表面下几微米 处进行表面处理形成表面压应力 ${ }^{[33]}$ 。表面相变引起 表面硬化可能会提高氧化锆样品的力学性能和耐磨
损性，但需考虑其相变层厚度，表面裂纹也会诱发 $\mathrm{t} \rightarrow \mathrm{m}$ 相变,一定程度的机械行为会引起表面晶粒析 出, 这对陶瓷而言是具有破坏性的 ${ }^{[34-35]}$ 。表面层机 械加工一方面可以提高材料的力学性能, 另一方面 因为相变、温度变化、加工程度导致材料有缺陷, 在 此过程中可通过获得尺寸较小且分布均匀的晶粒来 有效控制相变 ${ }^{[36]}$ 。表 1 为采用不同工艺制备的纳米 级 3Y-TZP 的晶粒尺寸。

成形后修复体需要进一步表面处理才能达到自 然牙齿的外观要求，而修复体的力学性能对表面处 理比较敏感。Chintapalli 等 ${ }^{[40]}$ 分析了喷砂过程对 3Y-TZP 机械性能的影响, 发现采用 $250 \mu \mathrm{m}$ 颗粒喷 砂陶瓷表面后，会产生分布不均的残余应力、空隙、 缺陷等, 致使其抗弯强度降低。Muñoz-Tabares 等 ${ }^{[41]}$ 发现研磨后表面出现横纹、再结晶及 $\mathrm{m}$ 相晶粒, 因 $\mathrm{m}$ 相颗粒尺寸小于相变临界尺寸，该过程抑制了相 变的产生, 抗弯强度由 $1197 \mathrm{MPa}$ 提高到 $1309 \mathrm{MPa}$ 。 研磨后的陶瓷在 $1200^{\circ} \mathrm{C}$, 保温时间 $1 \mathrm{~h}$ 环境中进行 退火处理, 可以使 $\mathrm{m}$ 相消失, 残余应力被去除, 表 面形貌恢复到表面处理前状态，如图 7(a)所示。观 察其横截面如图 7(b), 可以看出研磨后陶瓷表面横 纹也全部消失， 300 $\mathrm{nm}$ 等轴晶粒均匀分布于表层 下方。可见虽然研磨提高了材料的力学性能, 但后 续热处理对力学性能的提高并无大益。Inokoshi 等 ${ }^{[42]}$ 在此基础上分析了研磨、研磨后热处理、研磨后氧化 铝喷砂、研磨后 $\operatorname{CoJet}\left(\mathrm{SiO}_{2}\right)$ 喷砂等四种表面处理对陶 瓷力学性能的影响。由图 8 不同表面处理 $\mathrm{ZrO}_{2}$ 后的 Weibull 分布显示, 研磨后在 $1100^{\circ} \mathrm{C}$ 热处理 $30 \mathrm{~min}$ 的 $\mathrm{ZrO}_{2}$ 陶瓷易受到破坏，这是由于热处理过程降 低了表面残余应力，而研磨后再进行 $\mathrm{Al}_{2} \mathrm{O}_{3}$ 或 $\mathrm{SiO}_{2}$ 喷砂，因表面应力的增强，导致陶瓷的强度及断裂 㓞性均有提高。

若要获得具有较好服役性能的齿科 3Y-TZP 陶 瓷, 需要进一步研究其抗疲劳行为, 陶瓷的疲劳行 为通常采用静疲劳、动态疲劳、循环疲劳等方式来

表 1 不同方法制备 3Y-TZP 得到的晶粒尺寸

Table 1 Grain size of $3 Y-T Z P$ prepared by different methods

\begin{tabular}{|c|c|c|c|}
\hline Ref. & $\begin{array}{l}\text { Preparation } \\
\text { method }\end{array}$ & Preparation conditions & $\begin{array}{c}\text { Grain } \\
\text { size/nm }\end{array}$ \\
\hline [37] & Co-precipitation & $\begin{array}{l}\text { Sintering condition: } \\
1173 \mathrm{~K} \text { for } 10 \mathrm{~min}\end{array}$ & 3.3 \\
\hline [38] & $\begin{array}{l}\text { Vapor-phase } \\
\text { hydrolysis }\end{array}$ & $\begin{array}{l}\text { Precursor solution: } \\
\mathrm{ZrCl}_{4}: \mathrm{H}_{2} \mathrm{O}=1: 40\end{array}$ & 15.0 \\
\hline [39] & $\begin{array}{l}\text { Detonation } \\
\text { synthesis }\end{array}$ & Hot pressure moulding & 24.0 \\
\hline
\end{tabular}




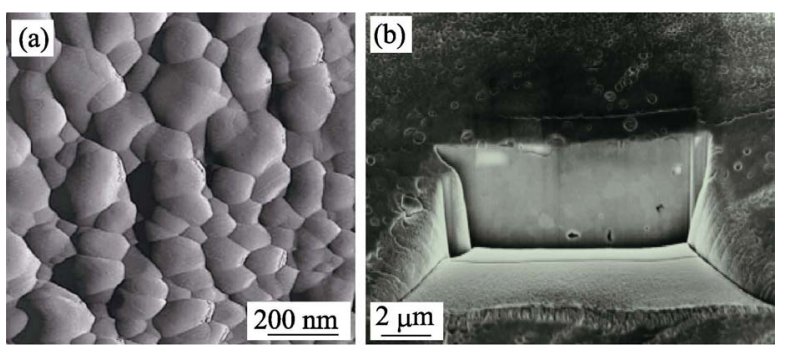

图 7 3Y-TZP 经过研磨并在 $1200{ }^{\circ} \mathrm{C}$ 退火 $1 \mathrm{~h}$ 后的形貌 ${ }^{[41]}$

Fig. 7 Surface of 3Y-TZP ground and annealed at $1200^{\circ} \mathrm{C}$ for $1 \mathrm{~h}^{[41]}$

(a) AFM; (b) FIB

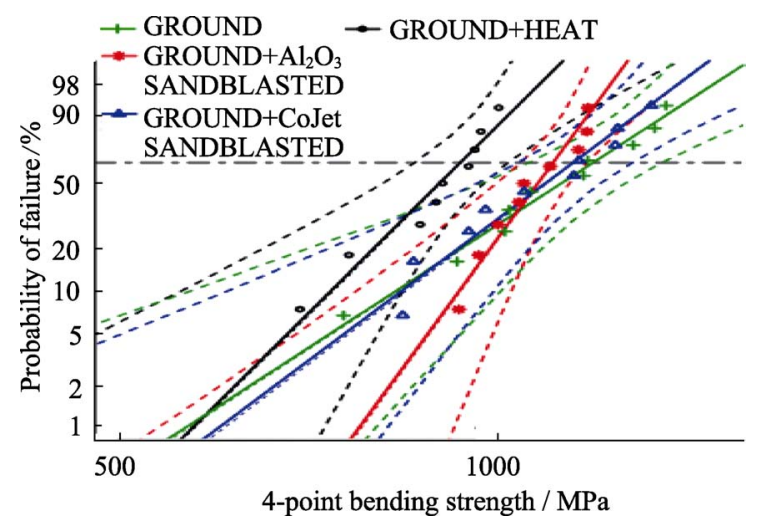

图 8 不同方法表面处理氧化锆后的 Weibull 分布情况 ${ }^{[42]}$

Fig. 8 Weibull analysis for the different surface treated Y-TZP ${ }^{[42]}$

表征。3Y-TZP 陶瓷因受到空气中水蒸气应力腐蚀作 用，在室温环境中会产生沿晶界扩展的慢裂纹，且 表面裂纹源产生慢裂纹的速率高于内部裂纹源, 因 此可通过提高表面压应力抑制表面慢裂纹的产生及 扩展, 从而提高其抗疲劳行为。Amaral 等 ${ }^{[43]}$ 研究了 3Y-TZP 表面处理后的疲劳行为, 结果表明经过 $30 \mu \mathrm{m}$ $\mathrm{SiO}_{2}$ 砂纸研磨后, 其疲劳强度、抗弯强度均有所提 高, 但随着疲劳循环次数 $\left(10^{2} \sim 10^{5}\right)$ 的增加, 陶瓷的 疲劳极限降低。

\subsection{In-Ceram}

In-Ceram 是通过注浆或软加工工艺制备而成的, 即在玻璃加入之前形成多孔陶瓷, 玻璃相的含量大 约占整个陶瓷成品的 $23 \%$ 。从 20 世纪 90 年代注浆 陶瓷开始应用于齿科修复，通过粉浆浇铸、烧结、 掺入镧系玻璃制备成多孔材料, 之后形成两个相互 关联的网状结构, 一个是玻璃相, 另一个是晶体相。 与传统注浆工艺相比, 软加工工艺使得陶瓷的大孔 隙率较低, 且孔径分布均匀, 因而应用愈来愈广泛。 目前有三种晶体比较适合: $\mathrm{Al}_{2} \mathrm{O}_{3} 、 \mathrm{MgAl}_{2} \mathrm{O}_{4}$ (镁铝尖 晶石)和 $12 \mathrm{Ce}-\mathrm{TZP}-\mathrm{Al}_{2} \mathrm{O}_{3}$ 。其中 $12 \mathrm{Ce}-\mathrm{TZP}-\mathrm{Al}_{2} \mathrm{O}_{3}$ 陶瓷是由 $34 \mathrm{vol} \% \mathrm{Al}_{2} \mathrm{O}_{3} 、 33 \mathrm{vol} \%$ 的 $12 \mathrm{~mol} \% \mathrm{CeO}_{2}$ 稳 定 $\mathrm{ZrO}_{2}$ 形成, 最终成品中含有 $23 \mathrm{vol} \%$ 玻璃相和 $8 \mathrm{vol} \%$ 残余孔隙率 ${ }^{[44]}$ 。由于双晶体增强体系 12
Ce-TZP- $\mathrm{Al}_{2} \mathrm{O}_{3}$ 中存在两种增韧机制, 使该陶瓷体系 相对于其他两种 In-Ceram 陶瓷具有较高的强度, 详 见表 $2^{[45]}$ 。

(1)氧化锆颗粒中的应力诱导相变会在相变颗 粒和周围玻璃基体中产生压应力, 并且随着微裂纹 的产生及扩展，颗粒周围产生拉应力，而氧化锆中 很难出现穿晶断裂, 这是其强化的主要原因。

(2)大颗粒氧化铝的出现会引起裂纹的偏转和 桥接。将这两种机制相结合就可以很好地解释为什 么氧化锆-氧化铝注浆陶瓷是所有注浆陶瓷中强度、 韧性最高的原因。

$12 \mathrm{Ce}-\mathrm{TZP}-\mathrm{Al}_{2} \mathrm{O}_{3}$ 因含有 $8 \mathrm{vol} \% \sim 11 \mathrm{vol} \%$ 的孔隙 率, 使其力学性能没有 3Y-TZP 好, 但比 3Y-TZP 具 有更好的热稳定性及抗低温老化性能。Chaar 等 ${ }^{[46]}$ 在修复完成后随访 10 年间, 评估结果显示 In-Ceram $\mathrm{ZrO}_{2}$ 固定义齿修复体(Fixed dental prosthesis, FDPs) 的存留率为 $93.16 \%$ 。

\subsection{Mg-PSZ}

Mg-PSZ 是以 $8 \mathrm{~mol} \% \sim 10 \mathrm{~mol} \% \mathrm{MgO}$ 作为稳定剂, 形成在立方相基体中含有四方相的一种氧化锆陶 瓷。其微观结构中 $40 \mathrm{wt} \%$ 四方相沉积在 $30 \mathrm{wt} \%$ 立方 相基体上，可以有效地抑制相变发生，即与 3Y-TZP 相比，其抗老化性能强，从图 9 Y-TZP、Mg-PSZ 老 化前后表面形貌，可以看出在相同的老化条件下， Mg-PSZ 表面并无结构损坏和晶粒析出现象 ${ }^{[47]}$ 。

表 2 不同类型 In-Ceram 陶瓷强度对比 ${ }^{[45]}$

Table 2 Strength comparison of different genres of In-Ceram ceramics ${ }^{[45]}$

\begin{tabular}{ccc}
\hline Crystal phase & Strength/MPa & Genre \\
\hline $\mathrm{Al}_{2} \mathrm{O}_{3}$ & $594 \pm 52$ & In-Ceram $\mathrm{Al}_{2} \mathrm{O}_{3}$ \\
Spinel & $378 \pm 65$ & In-Ceram Spinel \\
$12 \mathrm{Ce}-\mathrm{TZP}-\mathrm{Al}_{2} \mathrm{O}_{3}$ & $630 \pm 58$ & In-Ceram 12Ce-TZP- $\mathrm{Al}_{2} \mathrm{O}_{3}$ \\
\hline
\end{tabular}

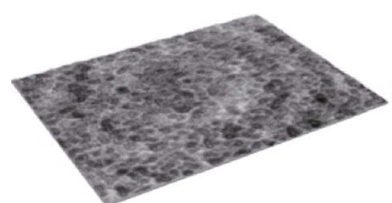

(a) Y-TZP, not aged

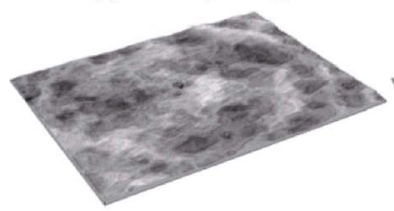

(c) Mg-PSZ, not aged

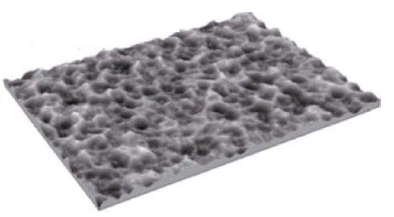

(b) Y-TZP, aged $49 \mathrm{~h}$

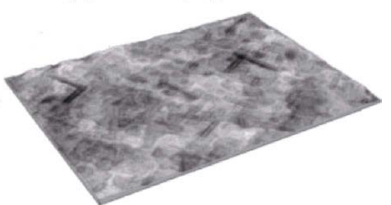

(d) Mg-PSZ, aged $49 \mathrm{~h}$
图 9 Y-TZP、Mg-PSZ 老化前后的表面形貌 ${ }^{[47]}$

Fig. 9 Surface topographies of (a) not aged Y-TZP, (b) aged Y-TZP, (c) not aged Mg-PSZ, and (d) aged Mg-PSZ ${ }^{[47]}$ 
但 Mg-PSZ 烧结温度通常在 $1800^{\circ} \mathrm{C}$ 左右, 因其 较高的烧结温度、粒径大(30 60 $\mu \mathrm{m})$ 及高孔隙率, 使 其应用没有 3Y-TZP 广泛 ${ }^{[48]}$ 。

\section{4 成形加工工艺}

1988 年 Duret 等 ${ }^{[49]}$ 首次将 CAD/CAM 技术引入 齿科修复领域。该技术最初仅用于完全烧结陶瓷的 表面处理, 1993 年开始用于对 $\mathrm{ZrO}_{2}$ 陶瓷进行机械加 工，目前已广泛用于部分烧结陶瓷的切削成形。

齿科氧化锆陶瓷通常采用两种成形工艺。

第一种工艺：坏体经过完全烧结后再进行切 削、磨削成形, 即硬加工 ${ }^{[50]}$ 。高温烧结后氧化锆陶 瓷体密度可达到理论密度的 $99 \%$, 但随后无论哪种 表面处理工艺都会引起表面发生不同程度的 $\mathrm{t} \rightarrow \mathrm{m}$ 相变, 在提高力学性能的同时也会诱导表面产生微 裂纹、降低材料可靠率等 ${ }^{[51]}$ 。Zhang 等 ${ }^{[52]}$ 提出一种 具有稳定且方便的超声振动磨削(Ultrasonic Vibration Assisted Grinding, UVAG)工艺, 作为一种新型的硬 加工技术引入口腔修复领域, 在一定程度上解决了 硬加工工艺对服役强度的影响。

第二种工艺: 为了避免硬加工工艺对服役强度 的影响, 采用 CAD/CAM 技术将部分烧结体切削成 形, 然后再进行完全烧结使氧化锆坏体获得最终的 机械性能, 即软加工 ${ }^{[50]}$ 。软加工过程节省了加工成 本, 提高了修复体精度, 减少了人工操作误差, 并 可实现远程加工及优势资源共享。在该过程中预烧 结温度对坏体的硬度、粗楉度影响比较大, 坏体处 理时需要一定的硬度, 但硬度过高不利于后期切削 加工，并且预烧结温度越高，其表面越粗糙。

目前, 对于哪一种工艺会获得更好的陶瓷修复 体还有争议。软加工的主要问题是软件需精确地将 框架在烧结过程中产生的线、体收缩与放大量相匹 配 ${ }^{[53]}$ 。当然与软加工相比, 硬加工操作时间长、切 削氧化锆烧结体比较困难, 而且会引起表面微裂纹 及缺陷等, 相比较而言软加工应用更为广泛。

\section{3 齿科氧化锆陶瓷韧性老化}

\section{1 氧化锆陶瓷韧性老化机理}

1981 年, Kobayashi 等 ${ }^{[54]}$ 首次发现低温老化现 象。当时一些研究者认为氧化锆在服役过程中很难 出现老化现象, 直到 2001 年大约 400 个股骨头在很 短时间内失效, 低温老化问题才开始真正受到大家 关注。

所谓低温老化(Low Temperature Degradation, LTD) 是在水或水蒸气的环境中, 表面缓慢发生 $\mathrm{t} \rightarrow \mathrm{m}$ 相 变, 之后材料表面因晶粒析出、结构损坏等原因使
其力学性能降低, 从而影响其服役时效, 即韧性老 化。氧化锆陶瓷在水中的老化机制如图 10 所示 ${ }^{[55]}$, 晶粒相变后因体积膨胀产生微裂纹, 微裂纹为水或 水蒸气的进入提供了通道, 图中红色路径代表由于 相变颗粒周围产生微裂纹导致水的进入。老化过程 中 $\mathrm{t} \rightarrow \mathrm{m}$ 相变量与老化时间的关系可由 JohnsonMehl-Avrami 公式表达 ${ }^{[15]}$ :

$$
f=1-\mathrm{e}^{-\mathrm{bt} \mathrm{n}^{\mathrm{n}}}
$$

式中 $f$ 相变分数, $t$ 为时间, $\mathrm{b} 、 \mathrm{n}$ 均为常量。可见老 化时间越长, 相变含量越高。

\section{2 氧化锆陶瓷韧性老化的影响因素及抑制 老化的措施}

齿科氧化锆陶瓷低温老化的影响因素主要包括: 晶粒尺寸、添加剂的含量、烧结温度、保温时间和 表面处理方式等。若要有效抑制氧化锆陶瓷老化, 提高其服役性能，可以通过控制晶粒尺寸、降低烧 结温度、缩短烧结时间、添加第二相和选择合适的 表面处理等。

Hallmann 等 ${ }^{[56]}$ 分析了添加剂及烧结温度对 Y-TZP 陶瓷老化行为的影响, 实验结果表明 Y-TZP 陶瓷 $1350^{\circ} \mathrm{C}$ 烧结 $2 \mathrm{~h}$, 晶粒尺寸小于 $0.3 \mu \mathrm{m}$, 老化实 验后并未发现 $\mathrm{m}$ 相、结构破坏、晶粒析出等现象。但 当烧结温度提高到 $1500^{\circ} \mathrm{C}$ 后, 晶粒尺寸大于 $0.3 \mu \mathrm{m}$, 水蒸气环境下明显出现老化现象, 如图 11(a), (b)所示, 随着晶粒尺寸变大、烧结温度升高, 材料表面结构有 明显损坏。同时在 $\mathrm{ZrO}_{2}$ 中添加一定含量的 $\mathrm{CeO}_{2}$ 、 $\mathrm{Fe}_{2} \mathrm{O}_{3}$ 后老化不明显, 说明添加剂有助于抑制老化 现象。Inokoshi 等 ${ }^{[57]}$ 也通过实验证明烧结温度越高、 保温时间越长, Y-TZP 和 In-Ceram 氧化锆陶瓷都越 容易老化。Cotic 等 ${ }^{[58]}$ 在此基础上认为在相同烧结条 件下, 晶粒排布越致密均匀, 其抗老化性能越好。

Zhang 等 ${ }^{[59]}$ 通过研究不同含量 $\mathrm{Al}_{2} \mathrm{O}_{3}$ 掺入 $2 \mathrm{~mol} \%$ $3 \mathrm{~mol} \% \mathrm{Y}_{2} \mathrm{O}_{3}-\mathrm{TZP}$ 对其低温老化性能的影响, 结果表 明: $\mathrm{ZrO}_{2}$ 晶界处 $\mathrm{Al}^{3+}$ 的存在会引起残余应力, 从而 有效抑制 Y-TZP 老化; $\mathrm{Al}_{2} \mathrm{O}_{3}$ 的添加量为 $0.25 \mathrm{wt} \%$

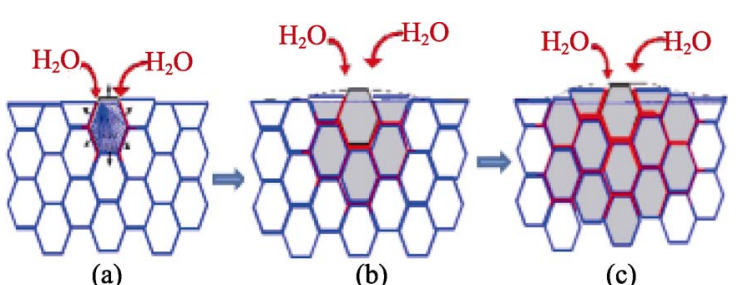

图 10 氧化锆在水中的老化过程 ${ }^{55]}$

Fig. 10 Scheme of the aging process ${ }^{[55]}$

(a) Nucleation on a particular grain at the surface, leading to microcracking and stresses to the neighbors; (b) Growth of the transformed zone, leading to surface roughening; (c) Further development of transformation 
时, 其抑制效果最佳, 且 $\mathrm{Al}^{3+}$ 在晶界处均匀分布, 如图 11(c)和(d)所示。Aragón-Duarte 等 ${ }^{[60]}$ 研究了不 同添加剂及其含量对氧化锆基陶瓷老化的影响程度, 详见表 3 , 从表中可以看出, 老化后硬度大小顺序 为: $8 Y-C S Z>3 Y-T Z P>A T Z, 8 Y-C S Z$ 老化实验前后 力学性能几乎没有变化。Huan 等 ${ }^{[61]}$ 采用固相合成法 制备 8Y-TZP、5Y-TZP, 进行老化实验结果发现 8Y-TZP 比 5Y-TZP 具有更加优良的抗老化性能, 因 此 8Y-TZP 将会成为未来生物医用领域的新材料。 除了在氧化锆基体中添加氧化物以外, Mohame 等 ${ }^{[62]}$ 通过实验发现在 3Y-TZP 中添加 CNTs 不仅可以 提高其力学性能, 还可以抑制其老化现象。

加工工艺及随后的表面处理对氧化锆基陶瓷老 化行为也有显著影响。Kim 等 ${ }^{[63]}$ 认为硬加工成形过 程中, 与传统的研磨、抛光等表面处理相比, 采用 $\mathrm{CAD} / \mathrm{CAM}$ 技术表面处理后氧化锆陶瓷更容易老化, 这是由于 CAD/CAM 加工对表面的损伤使烧结体表 面存在一定压应力。

除了控制晶粒尺寸、烧结温度、保温时间、改

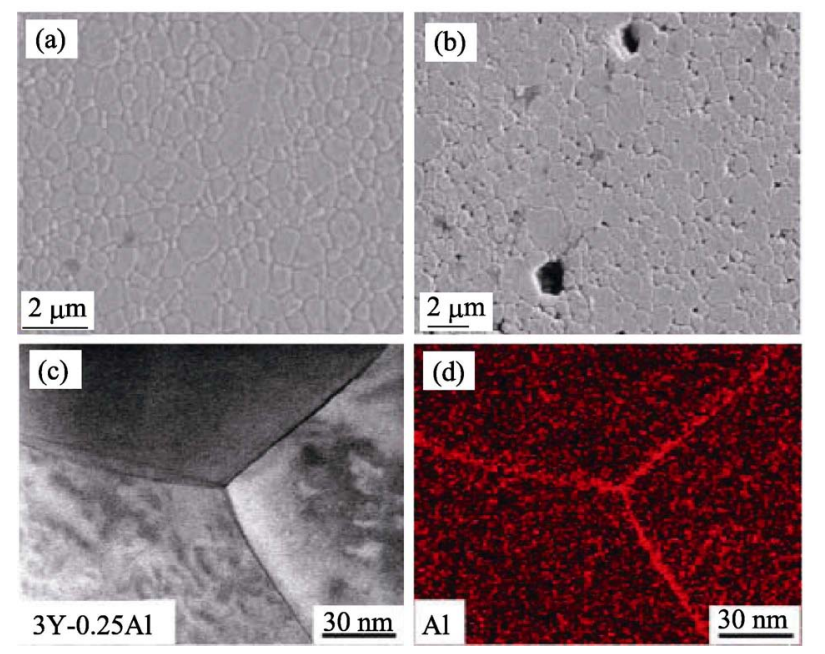

图 11 Y-TZP 老化(a)前(b)后表面形貌 ${ }^{[56]}$, (c)3Y-0.25Al 晶界 图, (d)3Y-0.25Al 中 $\mathrm{Al}$ 元素的分布 ${ }^{[59]}$

Fig. 11 Topographies of Y-TZP before (a) and after (b) aging ${ }^{[56]}$, STEM images of $3 \mathrm{Y}-0.25 \mathrm{Al}$ grain boundaries (c) and corresponding Al-distribution map (d) ${ }^{[59]}$

表 3 纳米压痕法评估陶瓷样品的力学性能 ${ }^{[60]}$

Table 3 Mechanical properties of the ceramics samples evaluated by nanoindentation ${ }^{[60]}$

\begin{tabular}{ccc}
\hline Ceramics & Hardness/GPa & $K_{\mathrm{IC}} /\left(\mathrm{MPa} \cdot \mathrm{m}^{1 / 2}\right)$ \\
\hline ATZ & $21 \pm 1.2$ & $4.2 \pm 0.1$ \\
ATZ with LTD & $12 \pm 1.5$ & $3.7 \pm 0.2$ \\
3Y-TZP & $25 \pm 0.8$ & $5.1 \pm 0.2$ \\
3Y-TZP with LTD & $15 \pm 1.5$ & $4.1 \pm 0.3$ \\
8Y-CSZ & $31.3 \pm 0.2$ & $3.77 \pm 0.02$ \\
8Y-CSZ with LTD & $31.2 \pm 0.3$ & $3.78 \pm 0.03$ \\
\hline
\end{tabular}

善表面处理工艺和添加第二相以外, 在氧化锆陶瓷 表面镀层也是一种减缓和抑制老化的新型工艺。 Hübsch 等 ${ }^{[64]}$ 采用物理气相沉积(PVD)技术, 将钛氧化 物 $\left(\mathrm{Ti}_{x} \mathrm{O}_{y}\right)$ 及钛氧化物－氧化铝 $\left(\mathrm{Ti}_{x} \mathrm{O}_{y}-\mathrm{Al}_{x} \mathrm{O}_{y}-\mathrm{Ti}_{x} \mathrm{O}_{y}\right)$ 透 明薄膜沉积在氧化锆基陶瓷表面, 实验证明涂层的 出现抑制了相变的进行, 从而抑制老化现象的发生。

\section{4 总结与展望}

本文概述了氧化锆基陶瓷在齿科领域的研究进 展, 总结了氧化锆陶瓷中存在的增韧机理, 列举了 目前齿科领域常用的 3Y-TZP、In-Ceram、Mg-PSZ 陶瓷, 并指出临床应用中氧化锆陶瓷存在的韧性老 化问题，在此基础上阐述了缓解或者抑制韧性老化 现象的因素和有效措施。虽然氧化锆陶瓷在齿科领 域的应用具有明显优势, 但目前仍存在以下问题:

1) 氧化锆陶瓷增韧机理较多, 但对不同增韧 机理间协同效应的研究还不够深入。

2) 氧化锆陶瓷的减法成形技术, 因刀具和材 料的直接接触, 会引起表面发生不同程度的 $\mathrm{t} \rightarrow \mathrm{m}$ 相变, 在促进力学性能提高的同时也会诱导表面微 裂纹的产生, 从而导致材料可靠性降低。

3) 相变产生的微裂纹, 会使材料在口腔环境 中更易出现晶粒析出、结构损坏等现象, 导致其服 役力学性能明显下降。

针对以上问题应在以下方面开展研究:

1) 加强对齿科氧化锆陶瓷协同增韧机理的深 入研究;

2) 引入氧化锆陶瓷的加法成形技术(3D 打印技 术 $^{[65-67]}$ ) 来彻底解决因刀具和材料的直接接触导致 的陶瓷表面损伤问题;

3) 以性能为导向, 加强材料设计研究, 选择新 型成形方法, 来提高氧化锆陶瓷的可靠性以及服役 寿命。

义齿是一种具有复杂外形的个性化的生物制品, 在极潮湿的口腔唾液等复杂的生物化学条件下服役, 承受咀嚼力、温度和 $\mathrm{pH}$ 值的频繁变化。随着人们 生活水平不断提高和保健意识持续增强, 人们对有 益人体健康的功能化义齿的需求会日趋迫切 ${ }^{[66-67]}$ 。 随着齿科氧化锆陶瓷综合力学性能的提高以及健康 功能化的未来需求, 其在生物医用领域的应用将会 越来越广泛。

\section{参考文献:}

[1] SOON G, PINGGUAN-MURPHY B, LAI K W, et al. Review of 
zirconia-based bioceramic: surface modification and cellular response. Ceramics International, 2016, 42(11): 12543-12555.

[2] FERNANDEZ-GARCIA E, CHEN X, GUTIERREZ-GONZALEZ C F, et al. Peptide-functionalized zirconia and new zirconia/titanium biocermets for dental applications. Journal of Dentistry, 2015, 43(9): 1162-1174.

[3] GARVIE R C, HANNINK R H, PASCOE R T. Ceramic steel? Nature, 1975, 258(5537): 703-704.

[4] DENRY I, KELLY J R. Emerging ceramic-based materials for dentistry. Journal of Dental Research, 2014, 93(12): 1235-1242.

[5] GARVIE R C, NICHOLSON P S. Phase analysis in zirconia systems. Journal of the American Ceramic Society, 2010, 55(6): 303-305.

[6] MAMIVAND M, ZAEEM M A, EL KADIRI H. Phase field modeling of stress-induced tetragonal to monoclinic transformation in zirconia and its effect on transformation toughening. Acta Materialia, 2014, 64: 208-219.

[7] WANG J, STEVENS R. Zirconia toughened alumina (ZTA) ceramics. Journal of Materials Science, 1989, 24(10): 3421-3440.

[8] MANICONE P F, IOMMETTI P R, RAAFFAELLI L. An overview of zirconia ceramics: basic properties and clinical applications. Journal of Dentistry, 2007, 35(11): 819-826.

[9] TORRICELLI P, VERNE E, BROVARONE C V, et al. Biological glass coating on ceramic materials: in vitro evaluation using primary osteoblast cultures from healthy and osteopenic rat bone. Biomaterials, 2001, 22(18): 2535-2543.

[10] TAN JIAN-GUO, ZHOU YONG-SHENG. Dental ceramic materials. Journal of Practical Department of Stomatology, 2009(7): 395- 397.

[11] HAN J, ZHAO J, SHEN Z. Zirconia ceramics in metal free implant dentistry. Advances in Applied Ceramics, 2017, 116(3): 138-150.

[12] DAUD M H M, ZENN Y H, ZAMAN J Q, et al. Evaluation of shear bond strength of a novel nano-zirconia and veneering ceramics. Ceramics International, 2017, 43(1): 1272-1277.

[13] MONTAZERIAN M, ZANOTTO E D. Bioactive and inert dental glass-ceramics. Journal of Biomedical Materials Research Part A, 2017, 105(2): 619-639.

[14] DEVILLE S, CHEVALIERR J, GREMILLARD L. Influence of surface finish and residual stresses on the ageing sensitivity of biomedical grade zirconia. Biomaterials, 2006, 27(10): 2186-2192.

[15] LAWSON S. Environmental degradation of zirconia ceramics. Journal of the European Ceramic Society, 1995, 15(6): 485-502.

[16] PALMERO P, FORNABAIO M, MONTANARO L, et al. Towards long lasting zirconia-based composites for dental implants. Part I: Innovative synthesis, microstructural characterization and in vitro stability. Biomaterials, 2015, 50: 38-46.

[17] EVANS A G, HEUER A H. Review-transformation toughening in ceramics: martensitic transformations in crack-tip stress fields. Journal of the American Ceramic Society, 1980, 63(5/6): 241-248.

[18] HANNINK R H J, KELLY P M, MUDDLE B C. Transformation toughening in zirconia-containing ceramics. Journal of the American Ceramic Society, 2000, 83(3): 461-487.

[19] KELLY J R, DENRY I. Stabilized zirconia as a structural ceramic: an overview. Dental Materials Official Publication of the Academy of Dental Materials, 2008, 24(3): 289-298.

[20] MCMEEKING R M, EVANS A G. Mechanics of transformationtoughening in brittle materials. Journal of the American Ceramic Society, 1982, 65(5): 242-246.

[21] AMAZIGO J C, BUDIANSKY B. Steady state crack growth in supercritically transforming materials. International Journal of Solids and Structures, 1988, 24(7): 751-755.

[22] ZHAO T, ZHU J, LUO J. Study of crack propagation behavior insingle crystalline tetragonal zirconia with the phase field method. Engineering Fracture Mechanics, 2016, 159: 155-173.
[23] SMIRNOV A, BELTRAN J I, RODRIGUEZ S T, et al. Unprecedented simultaneous enhancement in damage tolerance and fatigue resistance of zirconia/Ta composites. Scientific Reports, 2017, 7: 44922.

[24] LIU D, GAO Y, LIU J, et al. SiC whisker reinforced $\mathrm{ZrO}_{2}$ composites prepared by flash-sintering. Journal of the European Ceramic Society, 2016, 36(8): 2051-2055.

[25] ROBERTSON T, HUANG X, KEARSEY R. High temperature performance of mullite whisker-reinforced ZTA. Journal of Composite Materials, 2016, 50(26): 3719-3729.

[26] PECHARROMáN C, BELTRAN J I, ESTEBAN-BETEGON F, et al. Zirconia/nickel interface sinmicro-annano composites. Zeitschrift für Metallkunde, 2005, 96(5): 507-514.

[27] ZHANG LI-TONG, CHENG LAI-FEI. Discussion on strategy of sustainable development of ceramic matrix composites reinforced by continuous fiber. Journal of Composite Materials, 2007, 24(2): $1-6$.

[28] DUSZA J, BLUGAN G, MORGIEL J, et al. Hot pressed and spark plasma sintered zirconia/carbon nanofiber composites. Journal of the European Ceramic Society, 2009, 29(15): 3177-3184.

[29] 黄红燕. 牙科纳米氧化锆氧化铝 $\left(\mathrm{Al}_{2} \mathrm{O}_{3} / \mathrm{ZrO}_{2}\right)$ 复合陶瓷的基础 研究. 北京: 中国医科大学博士学位论文, 2009.

[30] NETTLESHIP I, STEVENS R. Tetragonal zirconia polycrystal (TZP)-a review. International Journal of High Technology Ceramics, 1987, 3(1): 1-32.

[31] PJETURSSON B E, SAILER I, MAKAROV N A, et al. All-ceramic or metal-ceramic tooth-supported fixed dental prostheses(FDPs)? a systematic review of the survival and complication rates. Part II: Multiple-unit FDPs. Dental Material, 2015, 31(6): 624-639.

[32] LE COADOU C, KARST N, EMIEUX F, et al. Assessment of ultrathin yttria-stabilized zirconia foils forbiomedical applications. Journal Material Science, 2015, 50(18): 6197-6207.

[33] BELLI R, GEINZER E, MUSCHWECK A, et al. Mechanical fatigue degradation of ceramics versus resin composites for dental restorations. Dental Material, 2014, 30(4): 424-432.

[34] PICONI C, MACCAURO G. Review: zirconia as a ceramic biomaterial. Biomaterials, 1999, 20(1): 1-25.

[35] ZHANG F, VANMEENSEL K, INOKOSHI M, et al. 3Y-TZP ceramics with improved hydrothermal degradation resistance and fracture toughness. Journal of the European Ceramic Society, 2014, 34(10): 2453-2463.

[36] AMARAL M, VALANDRO L F. Low-temperature degradation of a Y-TZP ceramic after surface treatments. Society for Biomaterial, 2013, 101(8): 1387-1392.

[37] HUANG H J, WANG M C. The phase formation and stability of tetragonal $\mathrm{ZrO}_{2}$ prepared in a silica bath. Ceramics International, 2013, 39(2): 1729-1739.

[38] WANG Q, LI C, GUO M, et al. Controllable synthesis of zirconia nano-powders using vapor-phasehy drolysis and the oretical analysis. Journal of Materials Chemistry A, 2014, 2(5): 1346-1352.

[39] KERN F, LINDNER V, GADOW R J CERAM. Low temperature degradation behaviour and mechanical properties of a 3Y-TZP manufactured from detonation synthesized powder. Journal of Ceramic Science and Technology, 2016, 7(4): 313-321.

[40] CHINTAPALLI R K, MESTRA R A, GARCIA M F, et al. Effect of sandblasting and residual stress on strength of zirconia for restorative dentistry applications. Journal of the Mechanical Behavior of Biomedical Materials, 2014, 29: 126-137.

[41] MUñOZ-TABARES J A, ANGLADA M. Hydrothermal degradation of ground 3Y-TZP. Journal of the European Ceramic Society, 2012, 32(2): 325-333. 
[42] INOKOSHI M, ZHANG F, VANMEENSEL K, et al. Residual compressive surface stress increases the bending strength of dental zirconia. Dental Materials, 2017. 33(4): 147-154.

[43] AMARAL M, CESAR P F, BOTTINO M A, et al. Fatigue behavior of Y-TZP ceramic after surface treatments. Journal of the Mechanical Behavior of Biomedical Materials, 2016, 57: 149-156.

[44] EL-GHANY O S A, SHERIEF A H. Zirconia based ceramics, some clinical and biological aspects: review. Future Dental Journal, 2016, 2(2): 55-64.

[45] DENRY I, HOLLOWAY J A. Ceramics for dental applications: a review. Materials, 2010, 3(1): 351-368.

[46] CHAAR M S, PASSIA N, KERN M. Ten-year clinical outcome of three-unit posterior FDPs made from a glass-infiltrated zirconia reinforced alumina ceramic (In-Ceram Zirconia). Journal of Dentistry, 2015, 43(5): 512-517.

[47] ROY M E, WHITESIDE L A, KATERBERG B J, et al. Phase transformation, roughness, and microhardness oartificially aged yttria-and magnesia-stabilized zirconia femoral heads. International Science, 2007, 83(4): 1096-1102.

[48] GARVIE R C, URBANI C, KENNEDY D R, et al. Biocompatibility of magnesia-partially stabilized zirconia (Mg-PSZ) ceramics. Journal of Materials Science, 1984, 19(10): 3224-3228.

[49] DURET F, BLOUIN J L, DURET B. CAD-CAM in dentistry. Journal American Dental Association, 1988, 117(6): 715-720.

[50] CARVALHO A O, BRUZI G, GIANNINI M, et al. Fatigue resistance of CAD/CAM complete crowns with a simplified cementation process. The Journal of Prosthetic Dentistry, 2014, 111(4): 310-317.

[51] GUAZZATO M, ALBAKRY M, RINGER S P, et al. Strength, fracture toughness and microstructure of a selection of all-ceramic materials. Part II. Zirconia-based dental ceramics. Dental Materials, 2004, 20(5): 449-456.

[52] ZHENG K, Li Z, LIAO W, et al. Friction and wear performance on ultrasonic vibration assisted grinding dental zirconia ceramics against natural tooth. Journal of the Brazilian Society of Mechanical Sciences and Engineering, 2016, 39(3): 833-843.

[53] ZARONE F, RUSSO S, SORRENTINO R. From porcelain fused to metal to zirconia: clinical and experimental considerations. Dental Material, 2011, 27(1): 83-96.

[54] KOBAYASHI K, KUWAJIMA H, MASAKI T. Phase change and mechanical properties of $\mathrm{ZrO}_{2}-\mathrm{Y}_{2} \mathrm{O}_{3}$ solid electrolyte after ageing. Solid State Ionics, 1981, 3(4): 489-493.

[55] CHEVALIER J. What future for zirconia as a biomaterial?
Biomaterials, 2006, 27(4): 535-543.

[56] HALLMANN L, ULMER P, REUSSER E, et al. Effect of dopants and sintering temperature on microstructure and low temperature degradation of dental Y-TZP-zirconia. Journal of the European Ceramic Society, 2012, 32(16): 4091-4104.

[57] INOKOSHI M, ZHANG F, DE MUNCK J, et al. Influence of sintering conditions on low-temperature degradation of dental zirconia. Dental Materials, 2014, 30(6): 669-678.

[58] COTIC J, JEVNIKAR P, KOCJAN A, et al. Complexity of the relationships between the sintering-temperature-dependent grain size, airborne-particle abrasion, ageing and strength of 3Y-TZP ceramics. Dental Materials, 2016, 32(4): 510-518.

[59] ZHANG F, VANMEENSEL K, INOKOSHI M, et al. Critical influence of alumina content on the low temperature degradation of 2-3mol\% yttria-stabilized TZP for dental restorations. Journal of the European Ceramic Society, 2015, 35(2): 741-750.

[60] ARAGON-DUARTE M C, NEVAREZ-RASCóN A, ESPARZAPONCE H E, et al. Nanomechanical properties of zirconia-yttria and alumina zirconia-yttria biomedical ceramics, subjected to low temperature aging. Ceramics International, 2017, 43(5): 39313939.

[61] HUAN C T, XIA J F, JIANG D Y, et al. Aging properties of yttriastabilized zirconia ceramics. Key Engineering Materials, 2012, 512-515: 435-438.

[62] MOHAMED E, TAHERI M, MEHRJOO M, et al. In vitro biocompatibility and ageing of 3Y-TZP/CNTs composites. Ceramics International, 2015, 41(10): 12773-12781.

[63] KIM J W, COVEL N S, GUESS P C, et al. Concerns of hydrothermal degradation in CAD/CAM zirconia. Journal of Dental Research, 2010, 89(1): 91-95.

[64] HÜBSCH C, DELLINGER P, MAIER H J, et al. Protection of yttriastabilized zirconia for dental applications by oxidic PVD coating. Acta Biomaterialia, 2015, 11(1): 488-493.

[65] TRUBY R L, LEWIS J A. Printing soft matter in three dimensions. Nature, 2016, 540(7633): 371-378.

[66] ZHU D B, XU A P, QU Y X, et al. Functionalized bio-artifact fabricated via selective slurry extrusion. Part 1: Preparation of slurry containing tourmaline superfine powders. Journal of Nanoscience and Nanotechnology, 2011, 11(12): 10891-10895.

[67] ZHU D B, LIANG J P, QU Y X, et al. Functionalized bio-artifact fabricated via selective slurry extrusion. Part 2: Fabrication of ceramic dental crown. Journal of Nanoscience and Nanotechnology, 2014, 14(5): 3703-3706. 